\title{
Introduction. De la victime à la victimisation : la construction d'un dispositif discursif
}

Introduction. From

victim to victimization: the construction of a discursive device

\section{Yana Grinshpun}

\section{(2) OpenEdition}

\section{Journals}

Édition électronique

URL : http://journals.openedition.org/aad/3400

DOI : 10.4000/aad.3400

ISSN : $1565-8961$

Éditeur

Université de Tel-Aviv

Référence électronique

Yana Grinshpun, «Introduction. De la victime à la victimisation : la construction d'un dispositif

discursif », Argumentation et Analyse du Discours [En ligne], 23 | 2019, mis en ligne le 18 octobre 2019, consulté le 28 novembre 2019. URL : http://journals.openedition.org/aad/3400 ; DOI : 10.4000/aad. 3400

Ce document a été généré automatiquement le 28 novembre 2019.

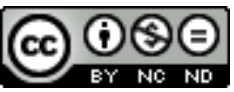

Argumentation \& analyse du discours est mis à disposition selon les termes de la licence Creative Commons Attribution - Pas d'Utilisation Commerciale - Pas de Modification 4.0 International. 


\title{
Introduction. De la victime à la victimisation : la construction d'un dispositif discursif
}

\author{
Introduction. From
}

victim to victimization: the construction of a discursive device

\author{
Yana Grinshpun
}

\section{Dispositif victimaire}

La meilleure façon de comprendre une époque, c'est de s'intéresser à ses obsessions. La nôtre est obnubilée par les victimes. Jamais autant d'attention n'avait été accordée aux souffrances d'autrui » (G. Erner, La société des victimes).

«Victime» et ses dérivés (victimaire, victimolâtre, victimiser) renvoient à des réalités socio-psycho-discursives très variées dont on trouve les manifestations dans un grand nombre de discours allant du discours ordinaire aux discours scientifiques ou juridiques. La dénomination "victime » couvre un large spectre de réalités sociales: des victimes du terrorisme aux victimes du photovoltaïque, en passant par les victimes des violences verbales, du racisme, du sexisme, de catastrophes naturelles, des maladies et même des pirates de l'attention (cf. Le Monde du 25.09.2017). Des méthodes et des enquêtes d'une certaine sociologie seraient construites "autour d'une conception victimaire ", l'histoire moderne construirait le «récit victimaire», le maniement du « registre victimaire » constituerait un moyen d'obtention de privilèges et des droits. Les associations de victimes jouissent de la reconnaissance institutionnelle et juridique et peuvent prétendre à une place dans l'espace public qui leur confère une reconnaissance sociale et un statut. Dans les débats actuels sur les identités, le mot «victime " s'entend avec une fréquence élevée dans les discours qui circulent sur la place publique. Se présenter comme victime ou être présenté comme telle par un tiers fonctionne comme un véritable argument dans un dispositif pluri-sémiotique qui poursuit des objectifs divers. 
2 La figure de victime s'inscrit dans l'histoire qui remonte, pour la culture occidentale, aux discussions théologiques du début du christianisme. Sa place et sa perception varient en fonction des contextes socio-historiques, des valeurs collectives et surtout des émotions que «la victime " provoque. La victime, dans cette perspective, apparait comme une construction sociale, élaborée par l'ensemble des discours, des représentations, des croyances et des émotions d'une époque donnée.

Dans ce numéro, nous voudrions insister particulièrement sur le fait qu'il s'agit dans la victimisation de constructions sous-tendues par des représentations sociales qui mobilisent des moyens discursifs et argumentatifs divers. Dans cette perspective, plusieurs pistes de réflexion se dégagent sur ce sujet :

1. Comment se construit le dispositif victimaire dans des types et des genres de discours différents? Quels sont les procédés argumentatifs utilisés pour sa mise en circulation, comment s'articulent les arguments de type rationnel et émotionnel dans ces dispositifs?

2. Le dispositif argumentatif est-il le même lorsqu'un groupe s'auto-victimise ou lorsqu'il est construit par un tiers qui lui, ne fait pas partie du groupe?

3. Si la place de la victime (de celui qui a souffert ou qui souffre) correspond aux valeurs positives collectives, comment un contre-discours arrive-t-il à se construire et à se justifier comme accusateur en disqualifiant le discours victimaire sans déroger aux valeurs communément admises dans une société donnée?

4. Quel impact a la circulation des discours antagonistes sur la «victimisation » sur les rapports de pouvoir et l'occupation des places dans le domaine social?

\subsection{Brève histoire de la figure victimaire}

Dans la perspective historique, il s'agit d'une position perçue de manière positive dans le système des valeurs de la société occidentale : être victime renvoie aux saints et aux martyrs, qu'il s'agisse de ceux qui sont morts pour la religion ou morts pour une idée.

Les saints et les martyrs ont toujours été objets d'admiration et de vénération après avoir été les objets de la piété. Les origines de la victimologie occidentale s'enracinent dans la tradition chrétienne pour laquelle la première victime absolue était Jésus, qui s'est sacrifié pour le salut de l'humanité. Cet héritage chrétien a laissé à la culture occidentale la conviction qu'il faut être du côté du faible, qui est censé susciter la compassion et à qui on doit la réparation de ses souffrances.

Pendant des siècles, les victimes n'ont pas été à l'ordre du jour. Le tournant dans la perception, et par conséquent, dans l'attitude à l'égard de cette figure sociale commence à la Révolution française, avec l'intérêt porté à la misère et aux changements des sensibilités collectives; il se poursuit à travers le 19

e

siècle avec l'avènement de nouveaux mouvements sociaux qui aspirent à transformer les conditions d'existence des acteurs sociaux considérés comme victimes des injustices de l'ordre social. 
La Première et la Deuxième Guerre mondiale et leurs victimes innombrables ne déclenchent pas une compassion semblable à celle que la société " pratique » aujourd'hui. Les premiers témoignages des rescapés des camps de la mort n'ont pas été entendus et il a fallu attendre les années 1960 et notamment Elie Wiesel pour que

l'Occident commence à parler des victimes de la Shoah (voir Jean-Michel Chaumont, La concurrence des victimes, pour plus de détails).

Le mouvement de reconnaissance du génocide juif en France a coïncidé avec le mouvement « révolutionnaire » de 1968 dont on peut dire qu'il s'opposait à l'ordre social et surtout à toute forme de pouvoir et d'autorité. Par conséquent, les rebelles de 68 manifestaient leur solidarité avec les opprimés que les nouveaux révolutionnaires cherchaient à faire apparaitre dans l'espace public. « Le souci de la reconnaissance de la souffrance des opprimés constituait un thème dominant plein d'analogie avec le thème de la victimisation et du manque de reconnaissance dont souffraient les victimes »

(Rimé 2015 : 114). Ces mouvements ont été rejoints par les féministes dans les années 1970. L'apogée de la reconnaissance de ces « nouvelles victimes » est marqué, entre autres, par l'ouvrage collectif dirigé par Pierre Bourdieu, La misère du monde(1993) à propos duquel Marcel Gauchet remarque :

Oubliée, la règle infrangible de traiter les faits sociaux comme des choses; place à la parole vive de l'acteur, à l'émotion du témoignage. Répudié, l'anti-humanisme théorique qui avait fourni le socle commun de la génération structuraliste et dont Bourdieu avait donné la version sociologique. C'est 1

'inhumanité

des logiques néo-libérales que l'enquête s'attache à mettre à nu, dans une science qui entend regarder la société non pas simplement d'en bas ou du côté des dominés,

Misère du Monde mais du point de vue de la victime. Tous ces déplacements font de la

l'exemple le plus réussi, le plus frappant, le plus significatif de l'ajustement du discours savant à la logique médiatique, à ses exigences identificatoires, à ses ressorts compassionnels (2002:317-318).

C'est par ces mouvements successifs que la victime se transforme en une catégorie sociale à part entière. Elle se construit dans le regard, et dans le discours de l'autre, qui joue un rôle important dans sa mise en fonctionnement social. La notion de victime, selon Erner $(2006: 10)$ « sert à désigner toute condition perçue comme insupportable par notre époque. Douleur physique, souffrance sociale ou psychologique, liée ou non à un traumatisme, une immense catégorie regroupe des individus dotés de destins profondément différents. » Erner, en sa qualité de sociologue, s'intéresse à une catégorie sociale, construite, qui ne possède pas de propriétés intrinsèques ou a priori

. Il manque, pourtant, à cette définition un paramètre important : ce n'est pas seulement une catégorie sociale, mais une catégorie socio-discursive, et même plus, une catégorie poly-sémiotique, produite par un ensemble de moyens discursifs et visuels à forte prépondérance émotionnelle. 


\subsection{Le rôle de l'émotion dans la construction victimaire}

La différence entre la victime « non-construite », réelle, pour ainsi dire, et celle qui est construite par les moyens discursifs et audio-visuels, réside dans l'intentionnalité. Les victimes qui revendiquent ce statut ou les médiateurs qui le font au nom des victimes

sont ceux qui peuvent et veulent se montrer souffrants en discours et en image. La monstration de la souffrance est supposée provoquer dans la société contemporaine le sentiment de compassion et, dans certains cas, d'indignation à l'égard des facteurs (humains ou non) qui ont amené la ou les personne(s) à l'état « victimaire ». L'état de « victime » crée une rupture dans l'ordre du monde, car ceux qui sont considérés comme victimes se trouvent dans une position inférieure à la normale. Quelque chose est dégradé de leur état et cette dégradation doit être réparée par ceux qui se trouvent dans la position supérieure, ceux dont l'intégrité n'a pas été touchée. Pour que les victimes bénéficient d'une réparation (la reconnaissance officielle de leurs souffrances, le châtiment des coupables de cette souffrance s'ils existent), il faut disposer

d'arguments pour faire adhérer les autres à la cause des victimes. L'argumentaire rationnel seul ne suffit pas, car il peut être réfuté par un contre-argument qui met en cause la validité de l'argumentation. En revanche, il est beaucoup plus difficile de réfuter ou d'invalider l'émotion. Par conséquent, l'argumentation « victimaire » ne peut pas se construire uniquement sur le logos

. Il faut que la souffrance soit mise en scène et qu'elle touche l'autre, qu'elle provoque un stimulus émotionnel pour que l'autre agisse.

R. Amossy rappelle dansL'argumentation dans le discours

(2012 [2000]) l'imbrication étroite des arguments rationnels et les arguments émotionnels. Elle donne en exemple l'indignation qu'on éprouve lorsque l'on voit persécuter des innocents. L'indignation peut en effet, avoir une base rationnelle, reposant sur une évaluation de la situation, mais elle peut également reposer sur la colère irraisonnée. Cette émotion peut être défendue par une argumentation qui s'appuie sur un système de valeurs et de croyances partagées par une société donnée.

Les analystes du discours et les spécialistes de l'argumentation qui s'y intéressent de près considèrent que les émotions sont structurées par des imaginaires socio-discursifs

(Charaudeau 2007) propres à chaque communauté socio-discursive. Elles émanent de

l'intrication étroite des discours et des valeurs partagées. Il ne s'agit donc pas d'une catégorie purement sociale ou purement psychologique. La manifestation des émotions, leur assimilation par le sujet social et leur perception dans une situation donnée sont codifiées par les normes morales et sociales en vigueur. Les émotions et leur expression varient selon les contextes socio-historiques, selon les normes culturelles dominantes. Comme l'Analyse du Discours ne dispose pas d'un appareil conceptuel et expérimental (mobilisé par la psychologie, par exemple) qui permette de mesurer une émotion ou de jauger son authenticité, c'est uniquement en tant que dispositif discursif qu'elle peut

être légitimement étudiée dans le cadre de cette discipline.

L'analyse du discours a pour objet d'étude le langage en tant qu'il fait sens dans une relation d'échange, qu'il est lui-même signe de quelque chose qui n'est pas dans lui et dont il est pourtant porteur. Dès lors, la

peur

, par exemple, n'est pas à considérer en fonction de la façon dont le sujet la manifeste par sa physiologie, ni comme une catégorie 
a priori

dans laquelle se mettrait le sujet selon ce qu'il est (ses propres tendances) ou selon

la situation dans laquelle il se trouve (seul face à un lion, au bord d'un précipice

etc.), ni comme le symptôme d'un comportement collectif (la panique), mais comme

signe de ce qui peut advenir au sujet

$\mathrm{du}$ fait que lui-même serait en mesure de le reconnaître comme une figure, comme un discours socialement codé... (Charaudeau 2000 : 74 ; c'est lui qui souligne).

2

La compassion n'a pas toujours ému (et mu) la communauté humaine. Hannah Arendt

Essais sur la Révolutionque ce sentiment est né au $18^{\mathrm{e}}$

siècle lorsque la misère et la pauvreté commencent à devenir objets des mouvements sociaux. La compassion serait liée, selon elle, à la conviction que le monde ne doit pas être comme il est.

Ce que l'Histoire nous enseigne, c'est que le spectacle de la misère qui inciterait les hommes à la pitié n'a rien d'une fatalité ; même durant les longs siècles au cours desquels le christianisme, religion de la miséricorde, déterminait l'échelle des valeurs morales de la civilisation occidentale, la compassion ne s'est exercée qu'en dehors de la hiérarchie ecclésiastique. Mais nous traitons ici des hommes du XVIII

e

siècle où cette indifférence séculaire était sur le point de disparaître et où, selon le mot de Rousseau, " une répugnance innée à voir souffrir un de nos semblables " s'était répandue dans certaines couches de la société européenne, et précisément parmi ceux qui firent la révolution française. Depuis lors, l'amour passionné de la compassion a hanté et guidé les êtres les meilleurs de toutes les révolutions...

(2012 : 105)

Et plus loin : 
Pour Robespierre, il allait de soi que la seule force qui pouvait et devait unir les différentes classes sociales en une seule nation était la compassion de ceux qui ne souffraient pas envers ceux qui étaient malheureux, la compassion des classes supérieures envers le petit peuple. La bonté de l'homme dans l'état de nature était devenue un axiome chez Rousseau qui découvrit que la compassion était la réaction humaine la plus naturelle face à la souffrance d'autrui et par conséquent le

fondement de tout rapport humain authentiquement naturel [...]. Si Rousseau introduisit la compassion dans la théorie politique, ce fut Robespierre qui la promut sur la place publique... (2012: 118-121)

La différence avec le $19^{\mathrm{e}}$

siècle est que le spectre de la compassion s'est élargi et la misère, la pauvreté et la souffrance sont perçues comme une offense au bien-être de l'humanité. L'époque romantique affine les esprits en matière de souffrance. Sans souffrance il ne pouvait y avoir de vérité, d'accès à la vraie vie. La souffrance permettait également de mettre en scène l'authenticité de celui qui s'adonnait à son exercice. On trouve le topique de la souffrance et de la vérité dans un grand nombre d'œuvres littéraires de la deuxième

moitié du 19

siècle : Novalis, Byron, Baudelaire, Dostoïevski. Les personnages mis en scène par ces auteurs ne sont pas victimes, bien au contraire, il s'agit souvent d'êtres héroïques qui souffrent d'être, qui portent sur leurs épaules l'incompréhension et le rejet du monde dans lequel ils se meuvent, en suscitant à la fois la compassion et l'admiration des lecteurs. En fait, on reste toujours dans la logique de la compassion envers la figure christique souffrante et innocente. Dans la victimologie moderne, cette place du Christ est toujours occupée. Malgré la sécularisation de la société à partir de la fin du 19

siècle, les figures christiques continuent à hanter l'espace public réel ou imaginaire (littéraire, philosophique). Cela va des Misérablesde Hugo à l'Idiotde Dostoïevski.

La compassion qu'éprouverait le lecteur/spectateur à l'égard de la victime mise en scène par les discours sociaux peut rester au stade du ressenti passif ou mener à l'action sociale. La compassion est susceptible de provoquer l'indignation. L'indignation est une émotion qui dépend étroitement des normes morales propres à chaque époque et à chaque lieu. C'est en elle que l'affect est inséparable d'une perception des valeurs et de jugements opérés au nom des valeurs partagées - ce qui en fait l'émotion politique, idéologique et médiatique par excellence. L'indignation collective doit ses quartiers de noblesses aux médias, qui à partir de la fin du $19^{\mathrm{e}}$ siècle la mettent en scène de manière régulière.

L'indignation telle qu'elle est dite, donnée à voir, mise en scène par les journaux, fonctionne donc comme une invitation au partage, une injonction à l'assentiment, un impératif éthique. Réelle ou fantasmée, elle devient via la publicité qui lui est faite, constitutive du lien moral et social. On ne brutalise pas les êtres innocents, on n'assassine pas. Il s'agit donc d'un sentiment qui fédère, qui crée du consensus, qui cimente des groupes, les mobilise et les pousse à la parole et à l'action, bref d'un sentiment (et à bien y réfléchir ils ne sont pas si nombreux) qui s'avoue, s'assume et s'actualise dans l'espace public. On le voit bien avec les foules indignées (AmbroiseRendu, Delporte 2005 : 15).

L'indignation est également une émotion parlante contrairement à beaucoup d'autres. Elle est souvent accompagnée de discours accusatoires ou exaltés qui s'appuient sur l'argumentation mêlant le rationnel avec l'émotionnel. L'indignation peut être 
provoquée par la compassion et entraîner une action sociale de réparation, de compensation symbolique, et quand c'est possible, de la punition des bourreaux. Selon L. Boltanski (1993:91),

dans l'indignation, la pitié est transformée. Elle ne demeure pas désarmée et, par conséquent, impuissante, mais se dote des armes de la colère. C'est en ce sens que l'on peut dire qu'elle pointe envers l'action puisque la colère [...] simule l'engagement dans une situation où elle pourrait s'accomplir en actes. Quelle serait leur nature? Bien évidemment de l'ordre de la violence. Mais à distance et, par conséquent, hors de tout contact physique, cette violence est condamnée à demeurer langagière. L'acte de parole qui la manifeste est une accusation. Ce n'est évidemment pas au malheureux lui-même qu'elle s'adresse. La transformation de la pitié en indignation suppose précisément une réorientation de l'attention, qui se détourne de la considération déprimante du malheureux et de ses souffrances pour aller chercher un persécuteur et se centrer sur lui.

La reconnaissance du statut de victime, suite aux discours indignés, donne dans certains cas, des droits d'exiger la réparation.

Pour le législateur, il ne s'agit pas davantage de blessés psychiques, mais d'individus lésés qui comme le définissent les Nations-Unis, « ont subi individuellement ou collectivement, un préjudice ». Or, qui dit préjudice, laisse place à la reconnaissance de droits spécifiques pour ceux qui l'ont subi et en particulier celui du droit à la réparation via une « indemnisation ». L'apparition du terme juridique «préjudice » dans le vocabulaire du XIXe siècle conduit simultanément à la récupération sociale de l'expression clivante de victime, du latin victima

: " créature vivante offerte aux dieux ». Le traumatisé, pour prétendre bénéficier d'une reconnaissance sociale de ce qu'il a vécu, est condamné à être victime s'il veut faire valoir ses droits (Romano et Cyrulnik 2015 : 57).

Il existe, en effet, des communautés, associations, collectifs de victimes dont certains ont aujourd'hui un statut légal. Les associations de victimes portent la parole des victimes auprès des pouvoirs publics et peuvent se constituer partie civile (http:// www.gouvernement.fr/guide-victimes/liste-d-associations-de-victimes).

\subsection{Emotions, victimes et médias}

L'émotion en tant que représentation collective est indissociable du fonctionnement des médias contemporains, elle y est omniprésente, car la construction de l'information est indissociable de la mise en scène du dispositif de captation. Il a été beaucoup dit et écrit sur les effets de dramatisation qui participent à la captation du lecteur/spectateur (voir notamment les travaux de P. Charaudeau 2002, 2006, 2013, D. Dayan 2006, J.-F. Tétu 2004). La nécessité de captation dans le discours médiatique lors de la présentation des évènements du monde peut se résumer par «la triade victime/ agresseur/sauveur. D'où trois types de discours: de victimisation, de portrait de l'ennemi, d'héroïsation » (Charaudeau 2006).

J.-F. Tétu souligne le rapport des médias avec l'émotion: "Il faut au support d'information quelque chose qui soit commun au groupe social : il doit représenter une situation à propos de laquelle une norme sociale (un jugement de valeur socialement partagé) met en cause dans un récit un actant qui est, en général soudainement, victime ou bénéficiaire $»^{3}$. du discours victimaire, nourrissent le dispositif médiatique de manière spectaculaire. 
La dramatisation du monde est primordiale pour le fonctionnement de l'appareil médiatique. Si on revient à l'indignation comme moteur d'action nécessaire pour changer l'état du monde dans lequel se trouve la victime, on s'aperçoit que c'est l'indignation qui constitue mieux que d'autres émotions l'événement médiatique. L'indignation accomplit le rêve d'évidence : elle se montre comme du "vrai » dans un discours univoque qui récuse tout doute et toute ambivalence du réel. Elle a un caractère subversif, mais aussi valorisant et valorisé. Elle active ou re-active des grandes catégories morales et les normes en vigueur dans une société donnée.

L'indignation qui met en scène la victime, participe pleinement à la mise en scène du monde. Les figures de compassion et d'indignation sont très médiatisées, car toutes les deux participent de la sensibilité collective telle qu'elle est définie et perçue par les médias. A son tour, le choix de diffuser, de rendre visible/ lisible/audible une situation imprégnée par la compassion ou par l'indignation agit sur la formation de la sensibilité collective. Il s'agit d'un processus en boucle. Le récit médiatique a besoin de figures ou de cas emblématiques où l'entité victimaire souffrante est prise en charge, et le mal s'il n'est pas puni, est nommé.

Le problème qui n'est abordé que d'une manière partielle dans ce numéro, mais qui mériterait d'être posée, c'est le choix opéré par les agents médiatiques des victimes intéressantes à mettre en scène, les victimes qui « rapportent » à la logique médiatique. Ce phénomène semble être lié l'histoire des sensibilités collectives et de leur évolution. Pour que ces sensibilités soient touchées, il faut susciter l'adhésion des consommateurs des médias pour déclencher le processus d'identification soit avec les victimes mises en scène, soit avec le discours indigné qui accompagne la mise en scène de la victime et qui fait appel aux normes collectives et valeurs communes enfreintes. Ne pas manifester l'adhésion à ces valeurs, ne pas afficher son identification avec les victimes ou avec les discours indignés peut devenir rédhibitoire et causer l'exclusion symbolique de l'indifférent de la communauté des valeurs.

L'exemple d'un tel fonctionnement est le début de «l'affaire Hamouri » en France. Un franco-palestinien demeurant à Jérusalem est accusé d'activités illégales en Israël et mis en réclusion. Une affaire inconnue du grand public, car inconnue aux médias. Jusqu'au moment où François Cluzet, un acteur français d'une renommée nationale, se montre très ému sur le plateau de France 2 par le fait que tout le monde ignore le sort "d'un type qui s'appelle Salah Hamouri ", et s'adresse à J.-F. Coppé, président de l'UMP, en lui reprochant de ne pas connaître l'histoire de Hamouri. Tapant à plusieurs reprises sur la table, Cluzet s'indigne, mu par la compassion pour son compatriote ${ }^{4}$. L'information qu'il donne de ce cas est inexacte, l'accusation imputée à Hamouri n'a rien à voir avec ce que dit Cluzet, qui énonce « Hamouri est contre la colonisation, c'est pourquoi on l'a mis en taule. Il n'a rien fait, il est donc innocent $»^{5}$. Ce n'est pas le "savoir » supposé de la cause d'arrestation de l'acteur, mais son émotion (l'indignation contre l'injustice infligée à un homme que l'acteur déclare innocent), qui va de pair avec sa notoriété médiatique, qui déclenchent « une recherche » ainsi qu'une série de reportages sur l'affaire Hamouri, et plus tard un documentaire.

C'est uniquement parce que la parole a été donnée à François Cluzet que la mise en scène de la "victime ", la création d'un " événement » et sa médiatisation est devenue possible. L'événement n'est pas constitué par le fait lui-même, mais par la réaction d'une célébrité qui apparaît à la fois comme citoyen témoin incarnant les valeurs partagées et comme revendicateur de la justice. La mise en scène des émotions 
télévisuelles, qui suscitent une série de reportages (voir le metteur en scène du film, Nadir Dendoune dans les premières minutes du film: "après le coup de gueule de François Cluzet, France 2 n'avait pas le choix ${ }^{6}$ que de faire un reportage enquête sur le sort de Hamouri »), est entièrement maîtrisée par l'instance médiatique. Ainsi, selon Charaudeau (2011: 164)

quel que soit le dispositif, c'est l'instance médiatique qui en a la maitrise totale imposant le point de vue selon lequel sera traité le thème, en reléguant les invités, qu'ils soient hommes politiques, experts ou simples citoyens-témoins, dans des rôles de faire valoir ou d'alibi : l'ensemble du dispositif est converti, volontairement ou non, en une machine à bloquer tout échange rationnel et explicatif sur la question traitée.

Les médias jouent ainsi un rôle prépondérant dans la construction de la place ou du statut de victime aujourd'hui, car ils permettent la médiation entre les victimes et les autres grâce à la parole du Tiers. Dans l'affaire Hamouri, il a fallu la voix d'un intermédiaire, entendue à la télévision, pour que l'affaire existe et pour que soit attribué à l'intéressé le statut de victime de l'injustice du gouvernement israélien. Le dispositif discursif victimaire doit être capable de déployer le récit, argumenté rationnellement et émotionnellement, des malheurs et des souffrances des victimes; récit qui doit toucher le récepteur, provoquer son émotion, lui permettant de ressentir son appartenance à la communauté des valeurs positives entérinées.

\subsection{Le rôle de l'image}

Il arrive souvent que la construction de la victime soit un processus pluri-sémiotique. Le rôle du discours est fondamental pour que la victime ne soit pas seulement montrée mais aussi instituée en tant que telle dans son statut social. Dans la société du spectacle, le rôle de l'image est également immense, surtout dans les médias contemporains. Les images ont un pouvoir performatif. Elles constituent une certaine preuve de la réalité et impliquent le déclenchement des émotions. Les images seraient constitutives de la mémoire collective en ce qu'elles produisent des sensations. Pour se figurer dans la mémoire collective, les images doivent apparaître régulièrement. Charaudeau appelle ces images « images-symptômes "; l'impact chez le spectateur est d'ordre émotionnel et psychologique. Une bonne illustration de ce phénomène est «l'affaire Jacqueline Sauvage » dont la grâce est due au rôle des médias qui ont contribué à sa constitution en victime, d'abord de son mari, puis de sa condition de femme, et ensuite du système judiciaire.

31 Le montage des images diffusé par l'hebdomadaire Le Point ${ }^{7}$, partie intégrante de l'article intitulé "Symbole des victimes de violence conjugale », des croquis faits dans la salle du tribunal, la musique, les photos des postures corporelles de la victime, celles des manifestants, la réception à l'Elysée par le président de la République, le résultat de l'action sociale - pétition signée par 400000 personnes -, tous ces événements (actions) permettent de mettre en scène une victime exemplaire dont la condition a provoqué un tollé créé par l'élaboration médiatique.

À la suite de sa condamnation, Jacqueline Sauvage est devenue un véritable symbole des victimes des violences conjugales subies par les femmes. Les collectifs féministes se sont approprié l'affaire et des manifestations ont été organisées dans Paris en décembre et janvier 2015. Une pétition a également été lancée pour appeler à sa libération, elle a réussi à rassembler plus de 430000 signatures. L'affaire a reçu le 
soutien de nombreuses personnalités, notamment Anne Hidalgo, maire PS de Paris, qui a créé un comité de soutien, avec l'ex-député écologiste européen Daniel Cohn-Bendit et le dirigeant du Front de gauche, Jean-Luc Mélenchon, réclamant la libération immédiate de Jacqueline Sauvage et la révision de la loi sur la légitime défense ${ }^{8}$. Il ne s'agit pas d'une simple victime, mais d'une victime symbole. Ce n'est pas tant la considération déprimante de l'accusée qui est importante ici, mais la focalisation sur les causes réelles ou supposées de sa souffrance.Comme le dit l'article duMonde, l'affaire est récupérée par les associations féministes et par la gauche socialiste. D'affaire judiciaire, elle se transforme en cause sociale, en lutte antisystème où la victime devient un symbole des luttes sociales.

\section{La victimisation comme contre-discours}

\subsection{Problèmes de définition}

La victimisation devient ainsi un phénomène social, psychologique et discursif caractéristique du fonctionnement de la société démocratique occidentale. Le passage $\mathrm{du}$ mot au concept, puis son ancrage dans l'univers discursif contemporain est un processus complexe, d'autant plus qu'il n'existe de critères objectifs de définition ni du mot ni du concept. Le mot semble renvoyer soit à un rapport problématique entre un groupe humain et la société toute entière ou entre deux ou plusieurs groupes. La « victimisation » est un mot récent. Dans le TLF, on trouve le verbe « victimiser » dans l'article consacré au verbe « victimer » (vieilli)

VICTIMER, verbe trans.

Vieilli

A. Maltraiter, soumettre à un traitement injuste. Sa joie et son plaisir Sont de faire souffrir. Mari que l'on victime, Amant, souffre-douleur (LABICHE, Frisette, 1846, 4, p. 223). - Part. passé en empl. adj. Et quels scrupules le retiendraient dans cette conquête? La morale? Il n'aperçoit autour de lui que dupeurs rapaces et dupes victimées (BOURGET, Essais psychol., 1883, p. 247).

B. - Empl. pronom. Faire de soi une victime, s'offrir comme victime. Il se signa et commença d'une voix douce un second discours sur le sacrifice, où il démontrait de quelle manière les Enfants du Carmel doivent se victimer, se sacrifier et détruire en eux-mêmes tous les faux prétextes de se soustraire à l'amour (BARRÈS, Colline insp., 1913, p. 201).

REM. Victimiser, verbe trans. Maltraiter, transformer en victime. J'en connais des tas qui ont perdu leur vie parce qu'on les a forcées ou parce qu'elles se sont crues forcées - à ce rôle [de veuves de guerre]. Pas question pour moi de me laisser "victimiser » deux fois: tu souffres assez comme ça sans en rajouter (Marie-Claire, sept. 1986, p. 293, col. 1). Prononc. et Orth. : [viktime], (il) victime [-tim]. Att. ds Ac. 1798.

Le Petit Robert indique que le terme vient de victimisation ou de l'anglais to victimize (1830) Transformer qqn en victime. Victimiser un accusé. - n. F. (1984) calqué de l'anglais victimisation. Certes, les définitions lexicographiques ne renseignent pas vraiment sur l'usage en langue commune et ne se rapportent pas forcément à quelque chose de défini dans le monde réel, cependant, on peut en tirer deux conclusions :

1. L'apparition du mot en français est assez tardive, datée des années 80.

2. La définition peut se prêter à l'ambiguïté : a) maltraiter quelqu'un, b) transformer quelqu'un en victime.

Dans le premier cas, il s'agit d'une maltraitance infligée à une personne, suite à laquelle elle se transforme en victime effective de l'acte délibéré, en d'autres termes, d'un acte 
de violence morale ou physique. Dans le deuxième cas, il s'agit d'un acte de transformation discursive d'un individu en victime. Autrement dit, la/les personne(s) «victimisée(s)» ne sont pas victimes d'un acte de violence effectif, mais se transforment en telle(s) suite au déploiement d'un dispositif discursif. Cela permet de supposer qu'entre l'acte de violence réel ou imaginaire et la personne ou le groupe humain, objet de cette violence, se déploie un dispositif socio-discursif qui rend possible leur construction et leur perception comme victimes. Dans les deux cas on a affaire à des victimes, mais dans le deuxième cas, il s'agit plus d'un statut ou d'une posture de " victime » qui est attribué au groupe par le garant discursif externe.

Il est très difficile de saisir la différence entre ces deux états du monde lorsque l'on a affaire à des énoncés sans leur inscription dans un contexte socio-historique particulier. Par exemple, la première définition donnée par Wikipédia comporte des paramètres psychologisants que nous ne sommes pas en mesure d'analyser faute d'appareil conceptuel approprié : " La victimisation est le fait d'être considéré comme étant particulièrementsensible à devenir victime d'un acte ou d'un phénomène donné. Par extension, le terme désigne la tendance à conférer aux victimes un statut social et une attention exagérée, proche http://fr.wikipedia.org/wiki/Victimisation). de la sacralisation » (

Il est impossible de savoir d'où provient cette définition, car l'article ne cite pas ses références, mais l'on voit déjà que le social et le psychologique s'y entremêlent. Elle ne semble pas très claire non plus peut-être à cause de l'emploi de l'adjectif « sensible » à

la place de "susceptible ». Néanmoins, quelque inexacte ou approximative soit la définition, elle permet de mettre en scène une dynamique socio-psychologique :

- avoir une « propension » à devenir victime

- acquisition du statut social de victime avec son bénéfice attentionnel.

L'emploi du syntagme « statut social » est remarquable, car il suggère l'existence d'un statut socialement reconnaissable, des droits liés à ce statut, et impliquerait certains comportements. En effet, le terme «statut » provenant de la sociologie implique une attente : on attend de la victime un certain type de comportement, une certaine manière d'être au monde. Cela va ensemble avec la présentation de soi (individuelle ou collective) et l'inscription dans des dispositifs discursifs particuliers.

\subsection{Victimisation comme type d'argument d'accusation}

Si la place de victime attribuée à celui qui a souffert ou qui souffre correspond à des valeurs collectives positives, si la victime provoque la compassion et peut susciter l'indignation des témoins de son malheur et de ses souffrances, pourquoi alors dénoncer ce statut ou cette place autour de laquelle se rassemblent tous ceux qui adhèrent à la communauté des valeurs ?

Lorsque l'on parle de victimisation, il s'agit de transformer quelqu'un (ou soi-même) en victime. La transformation implique le changement d'identité ou de statut: on se transforme en victime ou on transforme l'autre en victime moyennant des procédés de mise en scène des émotions, d'une élaboration des discours et d'une monstration des images qui provoquent la compassion et l'indignation. La souffrance des victimes, lorsqu'elle en vient à être publiquement reconnue, permet d'obtenir des droits, d'occuper une place au sein de l'espace public, de recevoir une visibilité et une certaine 
forme de pouvoir. La "victimisation» serait dans cette perspective une opération argumentée consciente (ou non) consistant à se présenter ou à être présentée par un tiers comme victime afin de bénéficier de droits, de reconnaissance, de bénéfices réels ou symboliques. La victimisation serait-ce alors une manière de tirer profit de la souffrance réelle ou imaginaire?

Pour répondre à cette question, il nous a semblé intéressant de voir des usages du mot « victimisation » dans le discours médiatique. Les faits sociaux sont inséparables de la circulation des discours et des dispositifs énonciatifs dont ils font partie. Dans le discours médiatique francophone, des commentateurs des blogs et des sites d'information l'utilisent comme un fait de langue " acquis », faisant partie de la langue commune. Les sites d'information montrent qu'il s'agit d'un usage élaboré, car victimisation est érigée en concept. « Victimisation » est constamment associée en tant que complément du nom aux substantifs faisant partie du champ sémantique d'élaboration consciente : « concept ", " discours ", « tactique », « technique », « stratégie », « idéologie », " posture » ou « mode » (modèle). Dans les locutions verbales, la « victimisation » est également " maniée », « jouée », « entretenue ", « vécue ». Elle fonctionne aussi comme thème des phrases averbales dans les slogans : « stop la victimisation », « marre de la » ou « halte à la » victimisation».

La victimisation est perçue comme un phénomène négatif, car elle est toujours préméditée, résultant d'une «stratégie », d'une « tactique » ou d'une « idéologie ». La place de la victime ou le statut de victime apparaît ainsi comme le résultat non pas de l'état réel de cette dernière, mais comme le fruit d'un ensemble de procédés prémédités menant à l'obtention de privilèges. Le récit de victimisation qui passe par la construction du discours victimaire (discours de victimisation) s'appuie sur des mécanismes argumentatifs divers, tant émotionnels que rationnels. La construction de ce discours implique les enjeux identitaires d'un groupe qui essaie de profiter de la place construite afin d'en tirer des bénéfices (réels ou symboliques). Enfin, l'accusation de victimisation se présente comme un contre-discours

dont l'objectif est de déconstruire, mettre à nu l'argumentaire des victimes afin de dénoncer une stratégie de manipulation de l'opinion publique, et les bénéfices obtenus grâce à la mise en scène de l' ethos

victimaire. Ce contre-discours peut, à son tour, engendrer un contre-discours où les accusés réfutent les arguments des accusateurs en les accusant à leur tour et en réitérant leur statut de victimes qui entraîne des droits. Ce que montre cet exemple, tiré d'un « manifeste » d'un " indigène de la République » :

On pourrait en rester là et affirmer fermement que nous refusons de tomber dans ce piège. Nous contenter de rappeler que nous ne nous

prétendons pas victimes, mais que nous le sommes

bel et bien, et que, parce que nous le sommes et en avons pleine conscience, nous refusons de nous complaire dans cette situation; que nous refusons de l'accepter comme notre sort inéluctable. Se dire victime, ce n'est ni geindre et se lamenter sur soi-même (

Et quand bien même! De quel droit ceux qui ne vivent pas les situations d'oppression reprochent-ils à ceux qui les vivent de se plaindre et de se lamenter -c'est-à-dire de verbaliser le tort qu'ils subissent, plutôt que de le subir en silence? 
) ; c'est, bien plus, se saisir des moyens de la transformation de son sort. Nous ne nous plaignons pas, nous n'implorons pas ; nous exigeons! Mais ne serait-ce pas cela que justement l'on nous reproche?

Cependant, à s'en tenir à ce propos nécessaire, nous négligerions sans doute un autre aspect. La victimisation a sa correspondance dans la culpabilisation. « Ne faites pas de victimisation », n'est-ce pas non plus une façon de dire : « Ne nous transformez pas en coupables »? Mais coupables de quoi ? S'il s'agit de reprocher aux générations actuelles les crimes de la traite, de l'esclavage, de la colonisation, cela ne peut être qu'un non-sens. Un livre saint a beau proclamer « Je punirais les crimes des pères sur les fils jusqu'à la 7ème génération ", nous n'avons, que je sache, pas fait nôtre ce précepte. (Gaudier 2005, http://lmsi.net/A-quoi-sert-lavictimisation)

Le topique victimaire sert d'argument au groupe qui est accusé de s'attribuer une position de victime (ce que les « indigènes » récusent en disant qu'ils ne prétendent pas

être, ils sont victimes des Blancs, des Juifs et de tous ceux qui n'adhèrent pas à leur point de vue). Le scripteur-énonciateur avance ici une contre-accusation qui consiste à recourir à une forme d'apodioxie, en disqualifiant l'adversaire accusateur par le refus d'argumenter au nom de l'évidence ontologique (être victime n'est pas prétendre être victime). Le locuteur renchérit sur le réel victimaire en créant une dichotomie entre les victimes qui ont subi des torts et tous ceux qui leur ont infligé les torts, au mépris de toute réalité historique (ni l'énonciateur ni les destinataires de ce discours n'étaient nés au moment des événements historiques qui ont causé les torts). En procédant par la prolepse, l'énonciateur construit une réfutation des arguments imaginaires qui lui permettent de dire que celui qui dénonce la position de victime s'attaque aux valeurs collectives, car celui qui n'a pas souffert ne peut éprouver les sensations qu'éprouvent les victimes. L'argument de la défense rationnalise l'émotion qui donnerait droit au

Il faut cependant faire la distinction entre les acteurs sociaux qui s'auto-victimisent, comme dans l'exemple précédent et d'autres groupe de victimes « vraies » qui sont

On pourrait décomposer l'état de choses de la manière suivante :

44 - Il ne s'agit pas d'un fait, mais d'une attitude, d'une stratégie, d'un complexe, d'un comportement qui implique deux positions : la position de la victime et la position du bourreau. Ces positions peuvent incomber à un individu ou à un groupe et peuvent être implicites ou explicites.

45 - Cette attitude ou cette stratégie, ce complexe ou ce comportement sont imputés à l'autre. Ils consisteraient à instrumentaliser la position de "victime » réelle ou construite pour en tirer des bénéfices réels ou symboliques. Parler de la victimisation c'est dénoncer le comportement d'instrumentalisation de la position de victime.

De ces exemples surgit une des propriétés essentielles de la victimisation: un mouvement par lequel ceux qui l'énoncent cherchent à disqualifier l'autre et cela tant par l'énonciation («sur un mode accusatoire ») que par l'énoncé : « la victimisation immunise le juif contre toute critique et immunise par là même Israël». Les dénonciateurs qui parlent de la victimisation construisent un contre-discours, un discours qui vise à instruire un procès d'intention prétendant démasquer le subterfuge de l'adversaire, l'accuser de vouloir tirer des bénéfices symboliques et réels de la place construite et auto-attribuée. Pour les accusateurs, le discours victimaire n'est pas perçu comme l'expression spontanée des souffrances mais plutôt comme une construction 
fondée sur un enchaînement d'arguments d'ordre émotionnel. Les contributions à ce numéro montrent de manière significative que dans le cas de la dénonciation de la posture de victime il s'agirait de dénoncer une construction à caractère argumentatif particulier, car elle viserait l'adhésion à sa cause par un dispositif argumentatif complexe où se croisent le pathos nécessaire pour provoquer l'adhésion et les arguments fondés sur la raison qui légitiment le dispositif discursif. L'accusation de victimisation met en scène la crainte que les bons sentiments auxquels l'émotion fait appel s'appuient sur des intentions inavouables.

\section{Présentation des articles}

Les contributeurs de ce numéro insistent particulièrement sur le fait que dans ces discours qui s'affrontent et dont l'un a pour l'objectif de disqualifier l'autre, il s'agit de constructions sous-tendues par des représentations sociales et par la mobilisation des moyens discursifs et argumentatifs disponibles. Les articles analysent des dispositifs victimaires très variés et abordent de multiples aspects de ces constructions sociodiscursives de plusieurs perspectives différentes.

Patrick Charaudeau propose une véritable cartographie de constructions victimaires de notre société en prenant soin de définir ce qu'on appelle le discours victimaire. La constitution de la personne ou d'un groupe de personnes en victime crée un état victimaire. La nature du processus par lequel cet état advient se fait voir dans le discours de victimisation. Dans les deux cas, on a affaire à un double rapport de subjectivité : d'une part, le rapport que le sujet entretient vis-à-vis du dommage subi et de l'agression, son rapport à la souffrance, d'autre part, le regard de l'autre, le point de vue de celui qui juge qui peut être qualifié comme victime. L'auteur montre que le processus de victimisation est indissociable de la problématique de l'émotion qui contribue à la construction de l'état victimaire en alliant affect et raison dans le discours. En développant les topiques pathémiques essentielles faisant partie des constructions discursives victimaires, Charaudeau élabore une grille d'émotions qui participent de la mise en discours de l'état victimaire.

Marianne Doury, en adoptant la perspective d'analyse de l'argumentation, s'interroge sur l'organisation argumentative et discursive des dispositifs déployés dans l'espace public après les attentats de janvier 2015. Il s'agit des discours de dénonciation du traitement inégal des victimes des attentats « deux poids deux mesures » qui feraient des victimes moins légitimes que d'autres, qu'elle appelle "victimes au carré». L'auteure exploite la manière dont la "règle de justice » théorisée par Perelman, est mobilisée par les discours visant à la fois la dénonciation d'une injustice et le rétablissement de la justice. Passant en revue un corpus important de réactions, Doury démontre que les positions construites par les discours argumentés pour désigner les victimes "vraies ou moins vraies" mobilisent des situations souvent fictives et s'appuient sur une rhétorique du ressentiment. Elle conclut que la désignation des types de victimes crées par ces discours contribuent moins à la restauration d'une forme de justice sociale qu'à l'extension de l'idéologie du ressentiment qui élargit la fracture sociale actuelle.

Pierre-André Taguieff, en s'appuyant sur une étude de l'accusation de meurtre rituel à l'encontre des Juifs, analyse la mise en place de la construction victimaire des haïsseurs des Juifs, qui a permis de justifier et de légitimer les mesures d'expulsion et 
d'extermination des Juifs depuis le douzième siècle. Il décortique les ressorts de «l'inversion victimaire » qui s'est produite depuis l'époque de l'antijudaïsme chrétien et qui ne cesse d'être rejouée dans les discours légitimant la violence contre les Juifs et aujourd'hui contre l'État hébreu. Ce phénomène obéit à un mécanisme rodé qui fonctionne toujours dans certains discours médiatiques et sur les réseaux sociaux : il met en scène l'autovictimisation d'un groupe (les haïsseurs des Juifs) à travers des récits criminalisant un autre groupe (les Juifs) qui est lui, innocent. Le philosophe élucide le scénario selon lequel les victimes imaginaires se substituent aux victimes réelles pour transformer ces dernières en responsables des malheurs réels ou potentiels du monde en leur attribuant des intentions criminelles. Une fois la figure du bourreau imaginaire créée et incrustée dans les représentations collectives, il devient légitime de se venger de lui en l'éliminant.

51 L'article de Sonia Branca aborde la problématique de l'autovictimisation et la construction du dispositif victimaire autour de la dichotomie Blancs/Noirs. L'auteure analyse la confrontation des discours des uns et des autres en se concentrant particulièrement sur le travail de redéfinition des concepts au sein des ensembles idéologiques adverses. Fondé sur l'articulation entre subjectivité énonciative et généralisation, le dispositif victimaire analysé contribue à la politisation des discours des entités qui se présentent comme victimes des pratiques sociales et des usages linguistiques. S. Branca montre que le dispositif victimaire s'appuie sur les émotions mises en récit et mises en scène dans l'acte du langage qui use de la violence verbale directe censé traduire les affects du groupe qui prétend être lésé dans ses droits. Elle analyse la dynamique discursive du sens du mot « nègre » et montre que son usage est corrélé aux positionnements identitaires des acteurs sociaux. Le sens du mot étant ambigu en français, ses connotations négatives sont utilisées pour la construction d'un dispositif victimaire à prétention antiraciste. Un tel dispositif fonctionne à l'instar du jeu de miroirs où les garants du contre-discours de ceux qui sont accusés de racisme se déclarent à leur tour victimes de leurs accusateurs.

Dans son texte, Eithan Orkibi fait écho à l'auteure précédente, en se focalisant sur la rhétorique de l'autovictimisation du premier ministre israélien Benyamin Netanyahu, qui en use en réponse à l'accusation de corruption. S'appuyant sur un cadre théorique inspiré par l'approche interactionnelle des études en victimologie, l'article examine trois dimensions constitutives du discours d'auto-victimisation de Netanyahou : l'appel à la pitié, les résonances culturelles et l'identité collective. L'analyse met en évidence les stratégies rhétoriques que l'auteur attribue au discours populiste dans la politique contemporaine. Même si l'étude porte sur un cas particulier du fonctionnement du discours auto-victimisant au sein du discours politique israélien, les procédés analysés peuvent être utiles pour l'étude du discours populiste contemporain en général. 
Laurène Renaut se focalise sur le discours de victimisation dans la communauté djihadiste en montrant comment ce dernier est mis au service d'une justification de

l'action violente. Elle remarque qu'une vision victimaire de l'identité personnelle soutient en fait la revendication d'une identité collective. La figure de victime apparait ainsi comme un facteur d'appartenance à un groupe qui s'auto-victimise et qui légitime

de ce fait le recours à la violence. L'auteure interroge l'impossible performativité du statut victimaire comme ressort du ressentiment et étudie le processus de « dévictimisation » visant autant à déshumaniser les « victimes » du terrorisme que les terroristes eux-mêmes, lesquels voient dans la promesse du martyre une forme d'héroïsation de la victime apparaissant comme une échappatoire au statut victimaire. Dans un deuxième temps, Renaut propose un panorama de différents types de contrediscours francophones (institutionnel, mémoriel et récits de repentis) qui s'attachent à combattre le discours de victimisation en se focalisant sur les stratégies discursives adoptées.

54 Une importante contribution de Marc Angenot ouvre la réflexion sur un type d'argumentation par le chiffrage des victimes. L'auteur montre par quels procédés cette argumentation se veut concluante dans les discours de dénonciation des régimes politiques à partir de la Révolution française et jusqu'au régime communiste. Dans cet article très documenté, Angenot analyse les usages historiques et politiques des chiffres de " pertes humaines ». Son étude est étayée par l'analyse minutieuse des polémiques successives sur les additions confrontées des victimes des totalitarismes du $20^{\mathrm{e}}$ siècle. Le chercheur rend compte de la manière dont l'argument par les chiffres, qui peut se passer de commentaires lorsqu'il s'agit d'extermination de masse par les régimes totalitaires, peut être détourné ou nié (dans le cas du négationnisme ouvert), par les défenseurs de ces régimes. Il analyse également la contre-argumentation qui consiste à accuser les dénonciateurs des régimes totalitaires de la haine de l'Idéal au nom duquel «les pertes humaines » sont indispensables, avançant que leur nombre est toujours exagéré, quelles que soient les sources.

Alain Rabatel part du constat que si la notion de victime est aujourd'hui valorisée, sa construction sociale tient compte du fait que les attitudes de victimisation et de recours systématique aux émotions d'apitoiement n'ont pas toujours bonne presse. L'auteur s'intéresse aux invariants des dispositifs victimaires, avant d'analyser la structure et la mise en discours particulière de ce qu'il appelle un « dispositif victimaire de combat». On pourrait dire qu'il s'agit ici d'un cas particulier de dispositif victimaire ou la victime ne se désigne pas comme telle. Rabatel étudie la « dernière lettre » d'Anne Bert, écrite avant sa mort. L'auteure, atteinte de sclérose latérale amyotrophique, revendique pour ceux qui sont dans la même situation qu'elle le droit d'être euthanasiés avec accompagnement médical pour mettre fin à leurs souffrances, en refusant tout autoapitoiement (ce qui rend son discours si singulier, car elle est une victime réelle d'une maladie incurable) et en choisissant des arguments différents selon les destinataires (ceux qui s'opposent à ses volontés, comme ceux qu'elle appelle à poursuivre son combat), au nom de l'intérêt général et de l'intérêt bien compris. 


\section{BIBLIOGRAPHIE}

Ambroise-Rendu, Anne-Claire, Chrisitian Delporte (éds) 2008. L'indignation. Histoire d'une émotion politique et morale. XIXe-XXe siècles (Paris : Nouveau Monde)

Amossy, Ruth. 2000. L'argumentation dans le discours (Paris : Nathan)

Arendt, Hannah. 2012 [1963] De la révolution (trad. Marie Berrane) (Paris : Gallimard)

Boltanski, Luc. 1993 La souffrance à distance (Paris : Gallimard, folio)

Bourdieu, Pierre. 1993. La misère du monde (Paris : Seuil)

Charaudeau Patrick. 2000. « Une problématisation discursive de l'émotion. A propos des effets de pathémisation à la télévision », Plantin C., M. Doury, V. Traverso V. (éds). Les émotions dans les interactions (Lyon : P.U.L.), 125-155

Charaudeau, Patrick. 2013. La conquête du pouvoir. Opinion, persuasion, valeur. Les discours d'une nouvelle donne politique. (Paris : L'Harmattan, coll. « Langue et parole)

Charaudeau, Patrick. 2006. «

Discours journalistique et positionnements énonciatifs. Frontières et dérives »Semen22, 29-44

Charaudeau, Patrick. 2007. «Les stéréotypes, c'est bien. Les imaginaires, c'est mieux », Boyer, H. (éd.). Stéréotypage, stéréotypes : fonctionnements ordinaires et mises en scène. (Paris : L'Harmattan), 49-64

Charaudeau, Patrick. 2008. "Pathos et discours politique", Rinn, M. (éd.), Émotions et discours. L'usage des passions dans la langue (Rennes : P. U. Rennes), consulté le 6 février 2019 sur le site de Charaudeau (Livres, articles, publications)

URL : http://www.patrick-charaudeau.com/Pathos-et-discours-politique,99.html

Charaudeau, Patrick. 2011.Les médias et l'information.L'impossible transparence du discours (Bruxelles : De Boeck)

Chaumont, Jean-Michel. 2002 [1997] La concurrence des victimes (Paris : La Découverte \& Syros)

Dayan, Daniel. 2006. La terreur spectacle. Terrorisme et télévision (Paris : INA \& De Boeck)

Erner, Guillaume. 2006. La société des victimes (Paris : La Découverte)

Gauchet, Marcel. 2002. La démocratie contre elle-même (Paris : Gallimard)

Gaudier, Christophe. 2005.Quelques éclaircissements sur un concept piégé,

http://lmsi.net/A-quoi-sert-la-victimisation(consulté le 16 août 2017)

Rimé, Bernard. (2015), Je suis victime. L'incroyable exploitation du trauma. (Helène Romano et Boris Cyrulnik éds) (Paris \& Savigny sur Orges : Duval), 109-134

Romano, Helène \& Boris Cyrulnik (éds). 2015. Je suis victime. L'incroyable exploitation du trauma (Paris \& Savigny sur Orges : Duval)

Tétu, Jean-François. 2004. «L'émotion dans les médias : dispositifs, formes et figures », Mots 75, $9-20$

\section{Dictionnaires}

Trésor de la Langue française en ligne http://atilf.atilf.fr/

Le Nouveau Petit Robert de la langue française 2008 


\section{NOTES}

1. Charaudeau 2008, p. 2 de l'article en ligne http://www.patrick-charaudeau.com/Pathos-etdiscours-politique.html)

2. On aurait pu évoquer la pitié, mais ce terme risque de comporter une nuance péjorative. G. Erner remarque à ce propos :

Le spectacle de la souffrance n'a pas toujours inspiré le même sentiment aux hommes. Aux époques où la religion organisait la société, Dieu accordait sa miséricorde aux malheureux. Les hommes, au mieux, pouvaient éprouver de la pitié, ceux qui les conduisait à offrir la charité à ceux que la Providence frappait cruellement. Mais dans une société laïque et démocratique comme la nôtre, où chaque homme voit en l'autre homme un semblable, la pitié n'a plus sa place.

Entre égaux une telle condescendance ajouterait à la douleur de l'injustice la souffrance de l'humiliation. La pitié a donc cédé la place à la compassion, une émotion provoquée par la douleur du semblable » (2006:11).

3. https://mots.revues.org/2843) article en ligne consulté le 12 septembre 2017

4. https://www.youtube.com/watch ?v =1FsS84aUSKg (consulté le 26 janvier 2019)

5. https://www.youtube.com/watch?v=1FsS84aUSKg

6. C'est nous qui mettons en italique

7. http://www.lepoint.fr/justice/francois-hollande-libere-jacquelinesauvage-28-12-2016-2093404_2386.php)

8. http://www.lemonde.fr/les-decodeurs/article/2016/12/29/pourquoi-l-affaire-jacquelinesauvage-fait-debat_5055435_4355770.html\#TeQYAbLqSRJRtfY1.99

\section{AUTEUR}

\section{YANA GRINSHPUN}

Université Paris III-Sorbonne Nouvelle 